\title{
Hiperplasia fibrosa em palato mole: relato de caso
}

Fibrous hyperplasia in the soft palate: a case report

Hiperplasia fibrosa en el paladar blando: reporte de un caso

Laís de Lima Barros Souza ${ }^{1 *}$, João Matheus dos Santos Silva ${ }^{1}$, Beatriz Santos Reis ${ }^{1}$, Mirian Karen Fernandes de Lima ${ }^{1}$, Karoline Gouvêa de Souza ${ }^{1}$, Maria Ítala Otacília Silva Barros ${ }^{1}$, Mykaelle Correia da Silva ${ }^{1}$, Jackson Manoel Diniz do Nascimento ${ }^{1}$, Arthur Eric Costa Wanderley ${ }^{1}$, Fernanda Braga Peixoto ${ }^{1}$.

\section{RESUMO}

Objetivo: Enfatizar a relevância diagnóstica na prática odontológica clínica, visando a correta conduta no tratamento da hiperplasia fibrosa em região de palato mole, bem como discutir a patogênese da lesão. Detalhamento do caso: Paciente do sexo feminino, 54 anos, feoderma, compareceu a Clínica Escola de Odontologia queixando-se de lesão assintomática que surgiu de forma lenta há aproximadamente 15 anos. Ao exame intraoral foi observada uma lesão nodular e pediculada em palato mole, de coloração normocrômica e violácea, superfície lisa, bem delimitada, de formato irregular e consistência amolecida, medindo $0.5 \times 1 \times 0.3$ centímetros. A hipótese de diagnóstico foi de hiperplasia fibrosa inflamatória. Optou-se pela biópsia excisional para confirmação da lesão através do estudo anatomopatológico. Considerações finais: A realização da biópsia é de extrema importância a fim de descartar outras hipóteses diagnósticas. É importante que o cirurgião-dentista esteja capacitado para propor o tratamento mais adequado, devolvendo o bem-estar do paciente e um prognóstico satisfatório.

Palavras-chave: Hiperplasia fibrosa, Palato mole, Patologia oral.

\begin{abstract}
Objective: To emphasize the diagnostic relevance in clinical dental practice, aiming at the correct conduct in the treatment of fibrous hyperplasia in the region of the soft palate, as well as discussing the pathogenesis of the lesion. Case details: Female patient, 54 years old, feoderma, attended the Dental School Clinic complaining of an asymptomatic lesion that developed slowly for approximately 15 years. On intraoral examination, a nodular and pedicled lesion was observed on the soft palate, with a normochromic and violet color, a smooth, well-delimited surface, with an irregular shape and softened consistency, measuring $0.5 \times 1 \times 0.3$ centimeters. The diagnostic hypothesis was that of inflammatory fibrous hyperplasia. An excisional biopsy was chosen to confirm the lesion through an anatomopathological study. Final considerations: The performance of the biopsy is extremely important in order to rule out other diagnostic hypotheses. It is important that the dental surgeon be trained to be able to propose the most appropriate treatment, restoring the patient's wellbeing and providing a satisfactory prognosis.
\end{abstract}

Keywords: Fibrous hyperplasia, Soft palate, Oral pathology.

\section{RESUMEN}

Objetivo: Enfatizar la relevancia diagnostica en la práctica odontológica clínica, con vista a una correcta conducta en lo tratamiento de la hiperplasia fibrosa en el paladar blando, así como discutir el patógeno de la lesión. Detalles del caso: Paciente femenino, 54 años, feoderma, llego a Clínica Escuela de Odontología quejándose de una lesión sin síntomas que apareció de forma lenta hace aproximadamente 15 años. El

${ }^{1}$ Centro Universitário Cesmac, Maceió - AL. `E-mail: laisbarrosl32@gmail.com 
análisis intraoral se observó una lesión nodular e pediculada en el paladar blando de coloración normocrómica y violáceo, superficie lisa, bien delimitada, de formato irregular y ablandada, midiendo $0.5 \times 1 \times 0.3$ centímetros. La hipótesis de diagnóstico fue de hiperplasia fibrosa inflamatoria. Se ha optado por una biopsia excisional para la confirmación de la lesión mediante un estudio histopatológico. Consideraciones finales: La realización de la biopsia es de gran importancia para excluir otras hipótesis diagnósticas. Es importante que el cirujano dentista esté capaz para proponer un tratamiento adecuado, llevando el bienestar al paciente e un pronóstico satisfactorio.

Palabras clave: Hiperplasia fibrosa, Paladar blando, Patología bucal.

\section{INTRODUÇÃO}

A mucosa oral quando exposta a traumas por longos períodos, está sujeita a uma série de lesões patológicas que podem se desenvolver em decorrência da ação de diferentes agentes etiológicos, resultando em uma maior incidência de lesões, principalmente hiperplásicas, onde classificam-se com base no tecido que as formam, podendo ser com predominância de tecidos de granulação (granuloma piogênico), tecido fibroso (hiperplasia fibrosa inflamatória - HFI) ou classificadas como outras lesões hiperplásicas (hiperplasia gengival induzida por fármacos) (SILVA MIP e DE OLIVEIRA MSB, 2019).

A hiperplasia fibrosa representa um processo proliferativo não-neoplásico, de origem inflamatória, proveniente de estímulos produzidos pela ação de agentes físicos, em geral traumas crônicos de baixa intensidade na mucosa, como: restaurações defeituosas, diastema, bordos cortantes de dentes e procedimentos iatrogênicos. A presença destes fatores desencadeia um processo inflamatório crônico, resultando na formação de tecido fibroso hiperplásico na região do trauma (QUEIROZ, et al., 2018; SILVA MIP e DE OLIVEIRA MSB, 2019). Ela pode apresentar outros sinônimos como: epúlide fissurada, epúlide por dentadura, tumor por lesão de dentadura ou hiperplasia fibrosa traumática. Entretanto, é melhor definida como hiperplasia fibrosa inflamatória (SANTOS CM e FERREIRA JRF, 2019).

Geralmente acomete o palato duro, e apesar da patogênese incerta, pode estar relacionada com maior frequência a próteses mal adaptadas. Além disso, na prática odontológica, comumente são observadas lesões orais decorrentes até mesmo da indevida orientação por parte do cirurgião-dentista ao paciente quanto o correto uso e higienização das mesmas, o que contribui para o surgimento de diversas patologias na cavidade bucal (SILVA MIP e DE OLIVEIRA MSB, 2019).

A má higienização oral, o uso de aparelho ortodôntico, maloclusão ou desequilíbrios hormonais, também podem estar relacionados com a formação da lesão. Além disso, é possível que sua etiologia também esteja associada a um transtorno de comportamento repetitivo, tal como o hábito de morder lábios e bochechas, iniciando o processo inflamatório e consequentemente a hiperplasia das células locais (FARYNOWSKA J, et al., 2018; SILVA MIP e DE OLIVEIRA MSB, 2019).

Esse tipo de lesão é mais prevalente durante a sexta década de vida, acometendo preferencialmente o sexo feminino, adultos de meia idade ou idosos. A respeito da localização, pode afetar qualquer área da mucosa oral, porém é mais frequentemente observada na região anterior da maxila e mandíbula e na região de fundo de sulco vestibular. Em relação a raça, há uma maior ocorrência em indivíduos leucodermas (BARROS RMG, et al., 2014).

Comumente, a lesão apresenta crescimento tecidual lento, de consistência flácida ou fibrosa à palpação, com coloração semelhante à mucosa adjacente e eritematosa. Possui base séssil ou pediculada, superfície lisa e pode ocorrer em vários sítios intraorais. Geralmente assintomática e quando sintomática os sintomas variam de dor leve a sangramento, ambos as custas do trauma recorrente (BARROS RMG, et al., 2014; DUTRA KL, et al., 2019).

Histopatologicamente, sua característica mais marcante é a hiperplasia do tecido conjuntivo fibroso. Geralmente seu epitélio de recobrimento é hiperparaqueratótico, com hiperplasia irregular das papilas. Raramente o epitélio apresenta hiperplasia papilar inflamatória ou epiteliomatosa (pseudocarcinomatosa) (CANUTO TSR, 2017; TRINDADE MGF, et al., 2018). 
De acordo com Barros RMG, et al. (2014), a presença de sinais displásicos no tecido epitelial das hiperplasias fibrosas inflamatórias caracterizam um conjunto de distúrbios na maturação celular, com potencial de malignização. A interpretação e observação da presença desses sinais displásicos podem mudar de forma significativa o diagnóstico, prognóstico e consequentemente o tratamento da referida lesão.

Apresentam-se como os principais diagnósticos diferenciais para este tipo de lesão o granuloma piogênico, lipofibroma, neurofibroma, rabdomioma, leiomioma, tumores de glândulas salivares menores e fibroma ossificante periférico (VAZ DA, et al., 2011; KUHN-DALL'MAGRO A, et al., 2013; BARROS RMG, et al., 2014).

Conforme a severidade e quantidade de tecido afetado pela lesão, pode-se definir o tratamento mais adequado. Lesões extensas necessitam de excisão cirúrgica com uma margem de segurança, e lesões pequenas podem ser tratadas de maneira conservadora. Para a remoção cirúrgica, três técnicas têm sido utilizadas: uso de bisturi convencional, elétrico ou remoção a laser. Antes de realizar a cirurgia é necessário que se diminua o componente inflamatório, com o objetivo de diminuir o sangramento pós-cirúrgico. Independente da técnica escolhida, é indispensável a eliminação do agente causador (CASIAN ROMERO A, et al., 2011; KUHN-DALL'MAGRO A, et al., 2013; SANTOS CM e FERREIRA JRF, 2019).

O objetivo do presente estudo foi relatar o caso clínico de uma paciente com 54 anos diagnosticada com hiperplasia fibrosa em região de palato mole, bem como discutir a patogênese da lesão e o método de tratamento proposto.

\section{DETALHAMENTO DO CASO}

O Termo de Consentimento para Fins Acadêmicos foi assinado pela paciente, consentindo o uso de dados da anamnese, exame físico, radiografias, modelos, desenhos, resultados de exames clínicos e laboratoriais ou quaisquer outras informações relativas ao diagnóstico, planejamento e tratamento que constituem seu acervo de dados pessoais para fins de ensino e aprimoramento científico.

Paciente do sexo feminino, 54 anos, feoderma, compareceu a Clínica Escola de Odontologia queixandose de lesão assintomática em boca. Ao ser questionada informou que surgiu há aproximadamente 15 anos, evoluindo de forma lenta.

Ao exame físico intraoral observou-se a presença de lesão nodular e pediculada em palato mole, bem delimitada, de coloração normocrômica e violácea, superfície lisa, formato irregular, consistência amolecida, indolor e medindo $0.5 \times 1 \times 0.3$ centímetros (Figura 1). Observou-se também a ausência dentária parcial em maxila e mandíbula e o uso de prótese provisória na maxila. As hipóteses de diagnóstico foram: granuloma piogênico, hiperplasia fibrosa inflamatória e pólipo fibroepitelial.

Figura 1 - Lesão nodular.

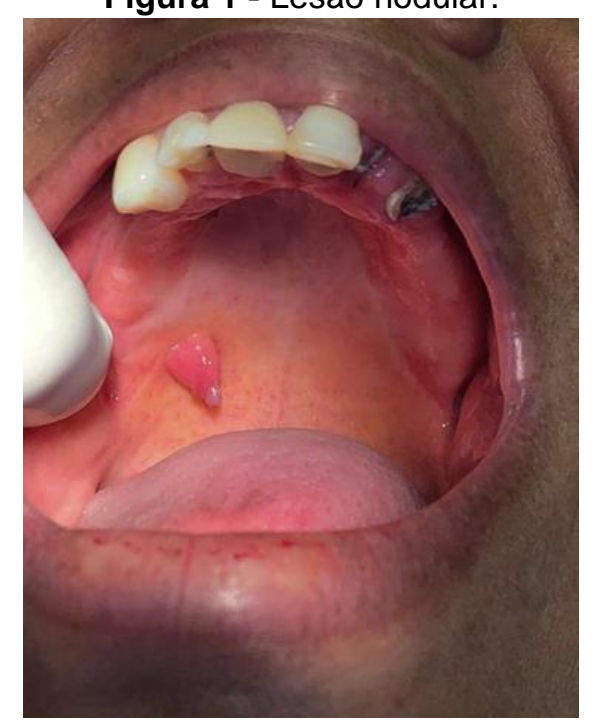

Fonte: Souza LLB, et al., 2021. 
Optou-se pela realização da biópsia excisional. Inicialmente sob anestesia local na região, foi feita uma incisão em forma de cunha com uso de bisturi convencional, removendo a lesão com pequena margem de segurança (Figura 2: A e B) e posteriormente foi realizada uma sutura simples com fio de nylon 3-0 (Figura 2: C).

Figura 2 - Remoção da lesão e sutura.

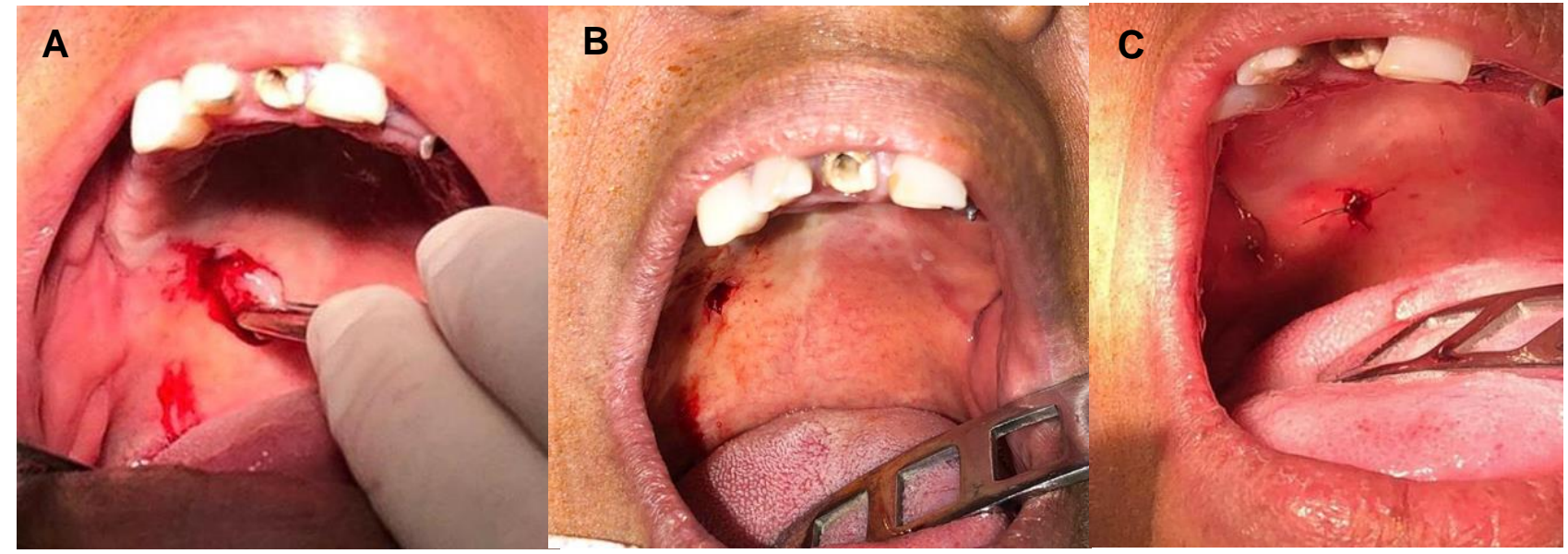

Fonte: Souza LLB, et al., 2021.

A peça cirúrgica foi armazenada e enviada para estudo anatomopatológico. O exame microscópico revelou fragmentos de mucosa oral revestidos por epitélio pavimentoso estratificado, e no tecido conjuntivo observouse intenso conteúdo de tecido fibroso (Figura 3: A e B). Diante disso, foi confirmada a hipótese diagnóstica de hiperplasia fibrosa.

Figura 3 - epitélio pavimento estratificado e tecido conjuntivo com intenso conteúdo de tecido fibroso, ambos indicados pelas setas azul e verde, respectivamente.
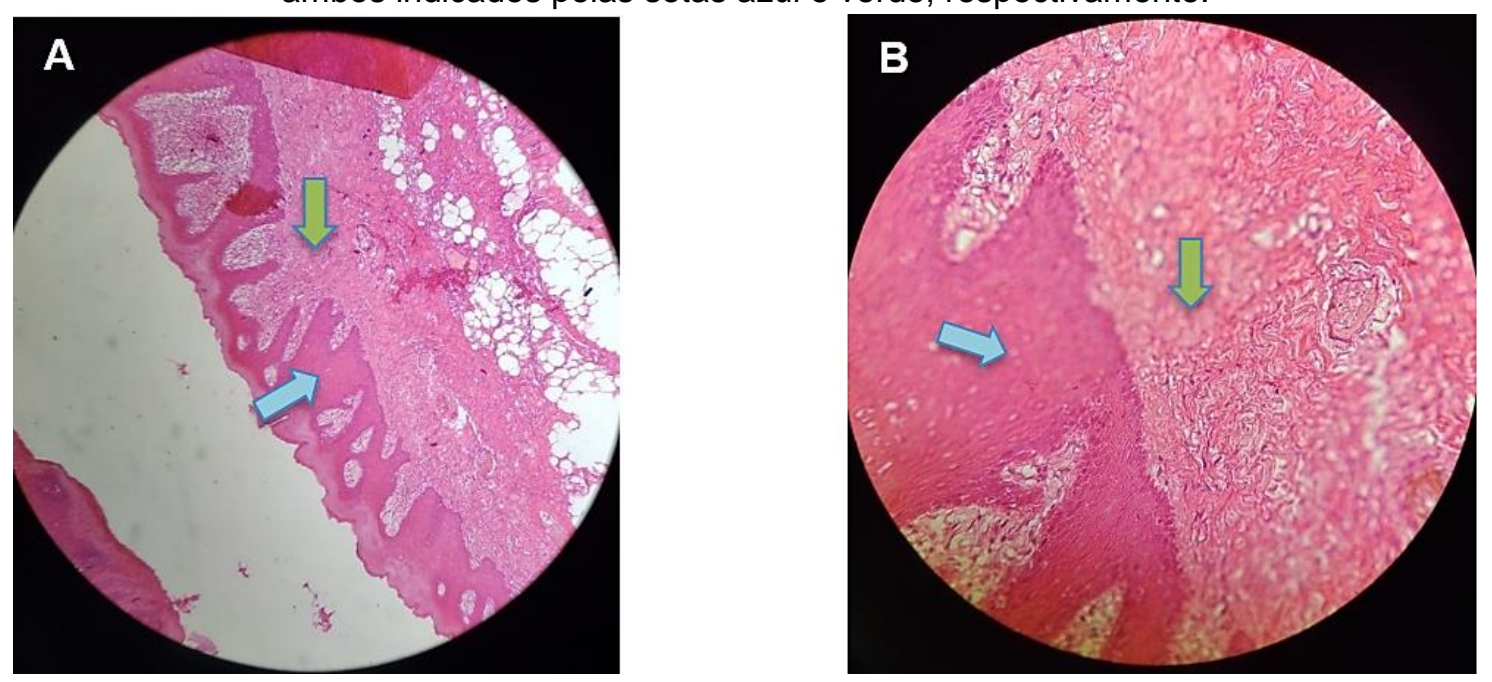

Fonte: Souza LLB, et al., 2021.

A paciente foi orientada sobre a necessidade de uma avaliação protética afim de confeccionar a prótese definitiva e evitar a recidiva da lesão, considerando que o fator causal fora removido. Após 15 dias a paciente retornou apresentando melhora do quadro e será mantida em acompanhamento para proservação.

\section{DISCUSSÃO}

A hiperplasia fibrosa inflamatória encontra-se entre as lesões mais frequentes decorrentes de traumas crônicos. É uma lesão de origem inflamatória proveniente de um crescimento tecidual fibroso, resultante de traumas mecânicos constantes e de baixa intensidade, causado principalmente por próteses mal adaptadas 
(MAGALHÃES ICR e GUEDES CCFV, 2020; SANTOS DPM, et al., 2021). É mais comum acometer adultos que fazem uso de prótese, podendo ser de crescimento lento e indolor. Todavia, pode ter outros fatores etiológicos como: diastemas, arestas de dentes cortantes, má higienização e manobras iatrogênicas (SILVEIRA EO, et al., 2020).

No caso em questão, a paciente apresentava uma lesão assintomática de crescimento lento, localizada em palato mole, que surgiu há aproximadamente quinze anos. A mesma fazia uso de prótese provisória mal adaptada na maxila, o que pode ter ocasionado o aparecimento da lesão, coincidindo assim com os relatos descritos nas literaturas (BARROS RMG, et al., 2014).

Autores como Santos DPM, et al. (2021) mostraram em seu estudo que cerca de $15 \%$ das patologias diagnosticadas predominam o sexo feminino na quinta e sexta década de vida, coincidindo com o exposto no caso clínico. Já a localização em palato mole diverge da maioria das literaturas pesquisadas, onde a localização pode acometer qualquer área da mucosa oral, sendo mais frequente em regiões como gengiva, bochecha, língua, lábios e palato, afetando geralmente a região anterior da maxila e mandíbula (BARROS RMG, et al., 2014; SANTOS DPM, et al., 2021). Todavia, de acordo com CANALI LGM, et al. (2020), os traumas por mastigação fazem com que o local de maior manifestação intraoral seja ao longo da linha oclusal da mucosa jugal, seguido pelo tecido gengival.

Alguns autores consideram que as mulheres se mostraram as mais predominantes no desenvolvimento da lesão, o que se dá, principalmente, devido à maior busca pela reabilitação funcional e estética por meio delas. O uso de prótese é um dos meios reabilitadores mais acessíveis, com excelentes qualidades, porém algumas limitações, tais quais podem influenciar diretamente no desencadeamento da patologia (MARTINS JC, et al., 2017; FARYNOWSKA J, et al., 2018).

A hiperplasia fibrosa inflamatória pode apresentar-se como um processo exofítico ou como um nódulo bem delimitado, diversificando de firme a flácida quando submetida ao trauma constante. Pode ser séssil ou pediculada, com coloração eritematosa ou normocrômica. $O$ crescimento é lento e na maioria dos casos ocorre de forma assintomática (CANALI LGM, et al., 2020). A lesão hiperplásica se apresenta, na grande maiorias dos casos, de tamanho pequeno, variando de 0,5 a 2 centímetros em sua maior dimensão (DUTRA $\mathrm{KL}$, et al., 2019).

A paciente apresentava uma lesão única, mole e pediculada, ratificando o encontrado na literatura, localizada em palato mole, de coloração normocrômica e violácea, com superfície lisa, limites nítidos, formato irregular, não infiltrativa e assintomática, assim como explanado por Canali LGM, et al. (2020). Medindo $0.5 \times 1 \times 0.3$ centímetros, o caso relatado vai de encontro aos achados literários relatados por Dutra KL, et al. (2019).

Por intermédio do exame histopatológico, a lesão é geralmente definida pela superprodução de tecido conjuntivo fibroso hiperplásico e da presença de numerosas células de fibroblastos e fibras colágenas, envoltas por epitélio pavimentoso estratificado com presença de acantose e eventuais áreas de atrofia (CANUTO TSR, 2017; DOCE DL, et al., 2019; CANALI LGM, et al., 2020). Após o envio da peça ao exame histopatológico, foi possível observar fragmentos de mucosa oral revestidos por epitélio pavimentoso estratificado, ratificando o exposto nas principais literaturas. Já no tecido conjuntivo, observou-se intenso conteúdo de tecido fibroso. Com isso, seu diagnóstico histopatológico foi de hiperplasia fibrosa.

Autores como Canali LGM, et al. (2020), relatam ainda o espessamento da camada de queratina (hiperqueratose) no tecido epitelial. Linfócitos e células plasmáticas, como leucócitos polimorfóides e alguns vasos sanguíneos, originam o infiltrado inflamatório crônico que está presente neste tipo de lesão. Ademais, por vezes podem estar presentes eosinófilos ou ocorrer à formação de folículos linfoides, não exibindo quadro de displasia, descartando a hipótese de lesão pré-maligna (CANUTO TSR, 2017; DOCE DL, et al., 2019; CANALI LGM, et al., 2020).

O tratamento baseia-se primeiramente na eliminação do agente causador, necessitando de uma reavaliação após um intervalo de 7 a 15 dias. Nos casos em que o conteúdo da lesão é predominantemente hemangiomatoso a lesão poderá regredir naturalmente, porém em casos fibróticos é necessário a remoção cirúrgica, com análise histopatológica. Alguns meios cirúrgicos são: remoção cirúrgica convencional com 
bisturi, remoção a laser e eletrocirurgia. Em casos de prótese mal adaptada, o protocolo deverá incluir a correção ou confecção de uma nova prótese para prevenir recidivas da lesão. (BATISTA VES, et al., 2013; NEVILLE BW, et al., 2016; DE SOUZA IT e SIMONATO LE, 2018; SANTOS DPM, et al., 2021).

Para realização de um adequado diagnóstico é de extrema importância o exame clínico do paciente, o qual deve ser constituído inicialmente pela anamnese bem detalhada, seguido pelo exame físico e exames complementares. Quanto a intervenção cirúrgica relatada no caso exposto, a biópsia excisional mostrou-se um exame complementar extremamente importante, visto que através do procedimento e análise anatomopatológica foi possível diferenciar a hiperplasia fibrosa de outras patologias que afetam a cavidade oral, obtendo um resultado de alta confiabilidade e um correto diagnóstico, a afim de propor o tratamento mais adequado ao paciente (DUTRA KL, et al., 2019; CANALI LGM, et al., 2020). A paciente segue orientada a respeito da necessidade de avaliação protética, manutenção da prótese e higiene bucal adequada para evitar recidivas da lesão.

A hiperplasia fibrosa representa um processo proliferativo não-neoplásico, causada na maioria dos casos por próteses mal adaptadas. Por se tratar de diagnóstico diferencial de outras lesões patológicas, é imprescindível a realização da biópsia, a fim de confirmar o diagnóstico. A atenção do cirurgião-dentista para as características clínicas, radiográficas e anatomopatológicas o auxiliarão na conclusão do diagnóstico, possibilitando oferecer o tratamento mais adequado, com prognóstico satisfatório, evitando a recidiva da lesão e devolvendo o bem-estar do paciente.

\section{REFERÊNCIAS}

1. SILVA MIP, OLIVEIRA MSB. Hiperplasia Fibrosa Palatina por Câmara de Sucção Tratada por Reembasador Resiliente - relato de caso. Dissertação (Graduação em Odontologia). Centro Universitário Unit, Maceió, 2019; 31p.

2. QUEIROZ, et al. Hiperplasia Fibrosa Inflamatória: relato de caso clínico. Anais da 19a Jornada Odontológica do Unifunec, 2018; 5(5).

3. SANTOS CM, FERREIRA JRF. Hiperplasia Fibrosa Inflamatória e Candidíase Oral Associadas ao Uso de Próteses Removíveis. Dissertação (Graduação em Odontologia). Centro Universitário São Lucas, Porto Velho, 2019; 23p.

4. FARYNOWSKA J, et al. Retrospective analysis of reactive hyperplastic lesions in the oral cavity. European Journal of Clinical and Experimental Medicine, 2018; 16(2): 92-96.

5. BARROS RMG, et al. Relato de Caso Clínico de Hiperplasia Fibrosa Inflamatória. Revista Odontológica de Araçatuba, 2014; 35(2): 15-18.

6. DUTRA KL, et al. Incidence of reactive hyperplastic lesions in the oral cavity: a 10 year retrospective study in Santa Catarina, Brazil. Brazilian Journal of Otorhinolaryngol, 2019; 85(4).

7. CANUTO TSR. Avaliação comparativa do uso do laser de nd:yag ou cirurgia convencional no tratamento de hiperplasia fibrosa inflamatória. Dissertação (Pós-graduação em odontologia) - Centro de Ciências da Saúde da Universidade Federal de Pernambuco. Recife, 2017; 86p.

8. TRINDADE MGF, et al. Lesões Associadas à Má Adaptação e Má Higienização da Prótese Total. Revista Multidisciplinar e de Psicologia, 2018; 12(42): 956-968.

9. VAZ DA, et al. Concordância entre os diagnósticos clínicos e histopatológicos do Laboratório de Patologia Bucal da Faculdade de Odontologia de Pernambuco. RPG Revista de Pós-Graduação, 2011; 18(4): 236-243.

10. KUHN-DALL'MAGRO A, et al. Laser cirúrgico no tratamento de hiperplasia fibrosa. RFO, 2013; 18(2): $206-210$.

11. CASIAN ROMERO A, et al. Hiperplasia Fibrosa Inflamatoria: reporte de un caso. Revista Clínica de Periodoncia, Implantología y Rehabilitación Oral, 2011; 4(2): 74-79.

12. MAGALHÃES ICR, GUEDES CCFV. Hiperplasia Fibrosa: Prevenção e Tratamento. Anais da Jornada Odontológica da FPM, 2020; 4(2): 1.

13. SANTOS DPM, et al. Hiperplasia Fibrosa Inflamatória em Mucosa Oral: relato de caso. Archives of Health Investigation, 2021; 10(2): 292-295.

14. SILVEIRA EO, et al. Acompanhamento, Procedência e Método Cirúrgico da Hiperplasia Fibrosa Inflamatória - relato de caso. Anais do I Fórum de Iniciação Científica de Odontologia da UNISC Santa Cruz do Sul, 2020; 1(1).

15. CANALI LGM, et al. Comprometimento de papila parotídea em lesão de hiperplasia fibrosa inflamatória: relato de caso. REAOdonto, 2020; 1: e4179.

16. MARTINS JC, et al. Prevalence of Oral Lesions Diagnosed at the ULBRA Canoas of Dental Diagnosis Service. Stomatos, 2017; 23(44).

17. DOCE DL, et al. Hiperplasia Palatina por Câmara de Sucção: relato de caso. Arch Health Invest, 2019; 8(11): 725727.

18. BATISTA VES, et al. Hiperplasia Fibrosa Inflamatória Ocasionada por Prótese Total Desadaptada: relato de caso. Revista Odontológica de Araçatuba, 2013; 34(2): 70-72.

19. NEVILLE BW, et al. Patologia Oral e Maxilofacial. 4nd ed, Rio de Janeiro: Editora Elsevier, 2016; 928p.

20. DE SOUZA IT, SIMONATO LE. Hiperplasia Fibrosa Inflamatória. Arch Health Invest, 2018; 7(4). 